\title{
Pseudo Zernike Moment and Deep Stacked Sparse Autoencoder for COVID-19 Diagnosis
}

\author{
Yu-Dong Zhang ${ }^{1}$, Muhammad Attique Khan', Ziquan Zhu ${ }^{3}$ and Shui-Hua Wang ${ }^{4, "}$ \\ ${ }^{1}$ School of Informatics, University of Leicester, Leicester, LE1 7RH, UK \\ ${ }^{2}$ Department of Computer Science, HITEC University Taxila, Taxila, Pakistan \\ ${ }^{3}$ Science in Civil Engineering, University of Florida, Gainesville, Florida, FL 32608, Gainesville, USA \\ ${ }^{4}$ School of Mathematics and Actuarial Science, University of Leicester, LE1 7RH, UK \\ ${ }^{*}$ Corresponding Author: Shui-Hua Wang. Email: shuihuawang@ieee.org \\ Received: 22 February 2021; Accepted: 07 April 2021
}

\begin{abstract}
Aim) COVID-19 is an ongoing infectious disease. It has caused more than $107.45 \mathrm{~m}$ confirmed cases and $2.35 \mathrm{~m}$ deaths till $11 / \mathrm{Feb} / 2021$. Traditional computer vision methods have achieved promising results on the automatic smart diagnosis. (Method) This study aims to propose a novel deep learning method that can obtain better performance. We use the pseudo-Zernike moment (PZM), derived from Zernike moment, as the extracted features. Two settings are introducing: (i) image plane over unit circle; and (ii) image plane inside the unit circle. Afterward, we use a deep-stacked sparse autoencoder (DSSAE) as the classifier. Besides, multiple-way data augmentation is chosen to overcome overfitting. The multiple-way data augmentation is based on Gaussian noise, salt-and-pepper noise, speckle noise, horizontal and vertical shear, rotation, Gamma correction, random translation and scaling. (Results) 10 runs of 10 -fold cross validation shows that our PZM-DSSAE method achieves a sensitivity of $92.06 \% \pm 1.54 \%$, a specificity of $92.56 \% \pm 1.06 \%$, a precision of $92.53 \% \pm 1.03 \%$, and an accuracy of $92.31 \% \pm 1.08 \%$. Its $\mathrm{F} 1$ score, MCC, and FMI arrive at $92.29 \% \pm 1.10 \%, 84.64 \% \pm 2.15 \%$, and $92.29 \%$ $\pm 1.10 \%$, respectively. The AUC of our model is 0.9576 . (Conclusion) We demonstrate "image plane over unit circle" can get better results than "image plane inside a unit circle." Besides, this proposed PZM-DSSAE model is better than eight state-of-the-art approaches.
\end{abstract}

Keywords: Pseudo Zernike moment; stacked sparse autoencoder; deep learning; COVID-19; multiple-way data augmentation; medical image analysis

\section{Introduction}

COVID-19 has caused more than $107.45 \mathrm{~m}$ confirmed cases and $2.35 \mathrm{~m}$ deaths till 11/Feb/2021 in about 192 countries/regions and 26 cruise/naval ships [1]. Fig. 1 shows the top 10 countries of cumulative confirmed cases and deaths, respectively. The main symptoms of COVID-19 are low fever, a new and ongoing cough, a loss or change to taste and smell [2]. In the UK,

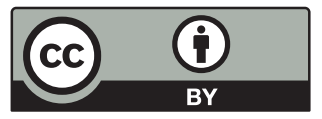

This work is licensed under a Creative Commons Attribution 4.0 International License, which permits unrestricted use, distribution, and reproduction in any medium, provided the original work is properly cited. 
three vaccines are formally approved as Pfizer/BioNTech, Oxford/AstraZeneca, and Moderna. Two COVID-19 diagnosis methods are available. The former is viral testing to test the existence of viral RNA fragments [3]. The swab test shortcomings are two folds: (i) the swab samples may be contaminated, and (ii) it needs to wait from several hours to several days to get the test results. The latter is chest imaging. There are two main chest imaging available: chest computed tomography (CCT) [4] and chest X-ray (CXR) [5].

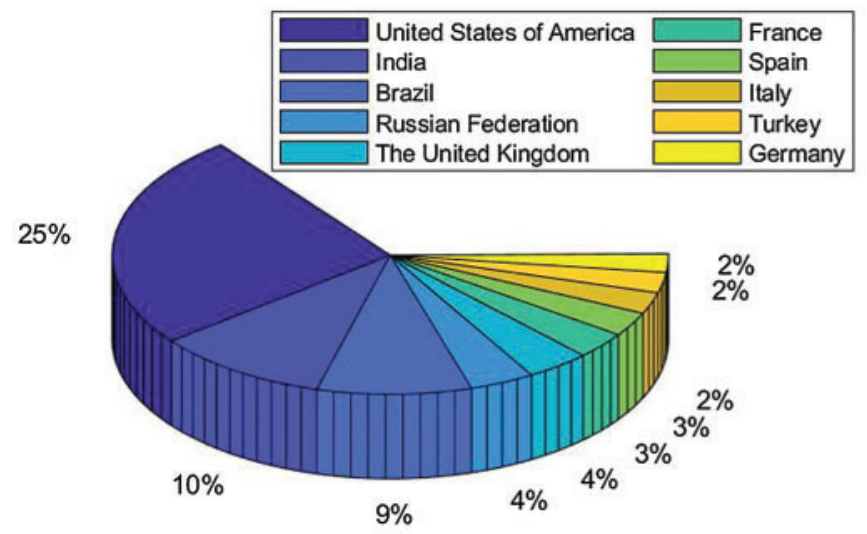

(a)

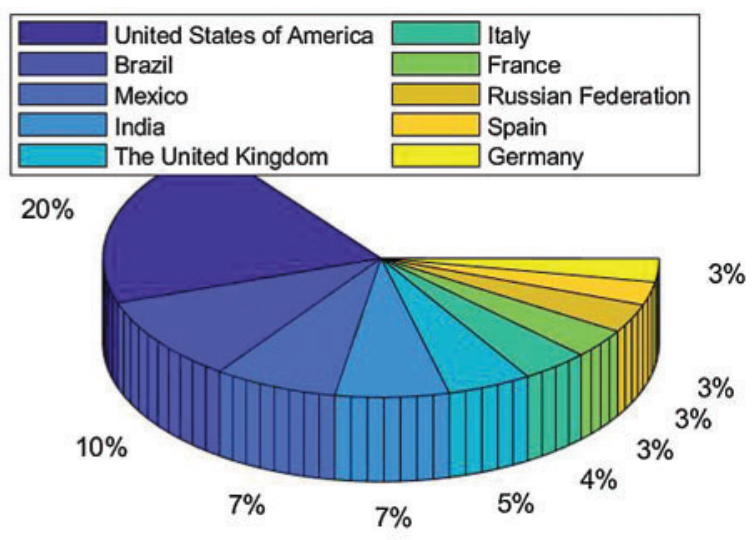

(b)

Figure 1: Data till 11/Feb/2021 (a) Cumulative confirmed cases (b) Cumulative deaths

CCT is one of the best chest imaging [6] techniques since it provides the finest resolution and can recognize extremely small nodules in the chest region. CCT employs computer-processed combinations of multiple X-ray observations taken from different angles [7] to produce highquality 3D tomographic images (virtual slices). In contrast, CXR only provides one 2D image, which performs poorly on soft tissue contrast. This study focuses on the CCT images [8].

Currently, numerous studies are working on using machine learning (ML) and deep learning (DL) technologies [9,10]. For example, Guo et al. [11] employed ResNet-18 for classifying thyroid images. Lu [12] utilized an extreme learning machine (ELM) trained by bat algorithm (BA). Those two approaches were not developing for COVID-19, but they can be transferred to the COVID-19 dataset easily and used as comparison basis approaches in our experiments. For COVID-19 researches, Yao [13] proposed a wavelet entropy biogeography-based optimization (WEBBO) method for COVID-19 diagnosis. Wu [14] presented three-segment biogeography-based optimization (3SBBO) for recognizing COVID-19 patients. Wang et al. [15] presented a DeCovNet. Their accuracy achieved 90.1\%. El-kenawy et al. [16] presented a novel feature selection voting classifier (FSVC) method for COVID-19 classification. Yu et al. [17] presented a GoogleNet-COD method to detect COVID-19. Chen [18] designed a gray-level co-occurrence matrix and support vector machine (GLCMSVM) method to classify COVID-19 images [19].

To further improve the performance of automatic COVID-19 diagnosis, this paper proposes a novel method that combines the traditional ML approach with the recent DL approach. We use the pseudo-Zernike moment (PZM) as the extracted features, and we use a deep-stacked sparse autoencoder (i.e., one of the deep neural networks) as the classifier. The combination achieves excellent results that overperform eight state-of-the-art approaches. The novelties of our paper lie in the following aspects 
- We are the first to apply a pseudo-Zernike moment to COVID-19 image analysis.

- Deep stacked sparse autoencoder (DSSAE) works better than traditional classifiers.

- Our proposed "PZM-DSSAE" model is better than eight state-of-the-art approaches.

\section{Dataset}

We use the dataset in reference [20], which contains 148 COVID-19 patients and 148 healthy control (HC) subjects. Slice level selection [20] was employed to generate $C_{1}=320$ COVID-19 images and $C_{2}=320 \mathrm{HC}$ images. The raw images are with sizes of $1024 \times 1024 \times 3$. A fourstep preprocessing was used on this dataset. First, the images are converted to grayscale to save storage amount. Second, histogram stretch is used to enhance the contrast. Third, border pixels are removed, which contains the text and ruler in the right side, and the check-up bed in the bottom. Finally, downsampling to width $W$ and height $H$ is carried out to further reduce the storage of the dataset. Fig. 2 display one example of COVID-19 patient and one sample of HC subject. Algorithm 1 itemizes the pseudocode of preprocessing.

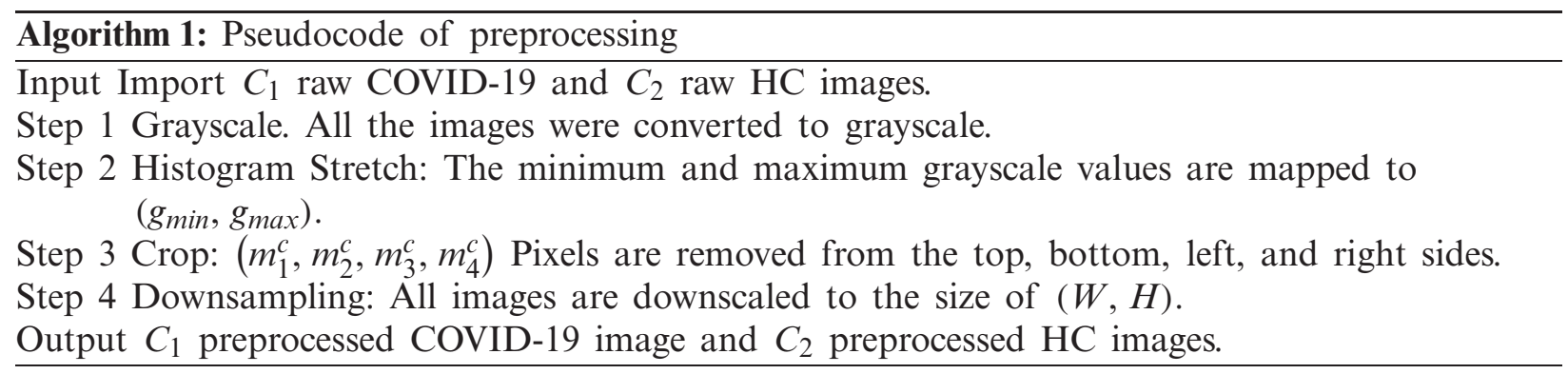

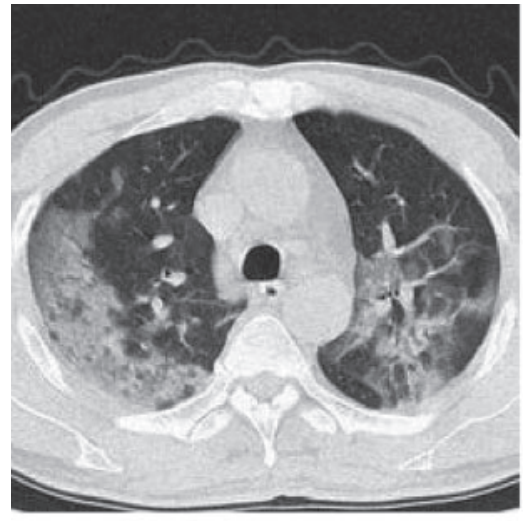

(a)

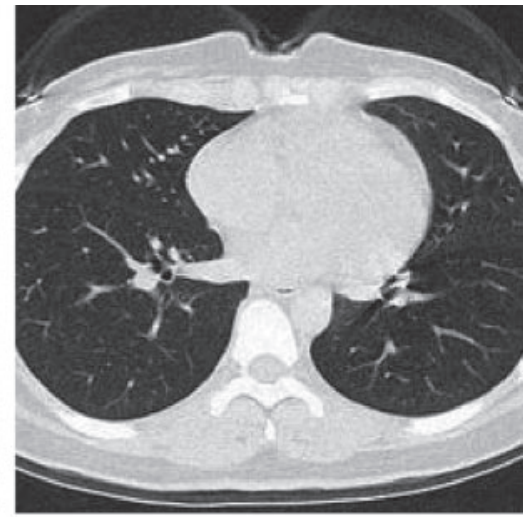

(b)

Figure 2: Example of preprocessed images (a) COVID-19 (b) HC

\section{Methodology}

\subsection{Pseudo Zernike Moment}

Tab. 1 displays the abbreviation list Image moment was firstly introduced by $\mathrm{Hu}$ [21], who used geometric moments to generate a set of invariants. Hu's moments have been widely used 
in knee osteoarthritis classification [22], brain tumor classification [23], etc. However, geometric moments are sensitive to noise. Thus, Teague [24] introduced Zernike moments (ZMs) based on orthogonal Zernike polynomials. The orthogonal moments have been proven to be more robust in noisy conditions, and they can achieve a near-zero value of redundancy measure [25].

Table 1: Abbreviation list

\begin{tabular}{llll}
\hline Abbreviation & Meaning & Abbreviation & Meaning \\
\hline AE & Autoencoder & HC & Healthy control \\
AUC & Area under the curve & IP & Image plane \\
CCT & Chest computed tomography & MCC & Matthews correlation coefficient \\
CM & Confusion matrix & ML & Machine learning \\
CXR & Chest X-ray & PZM & Pseudo Zernike moment \\
DL & Deep learning & ROC & Receiver operating characteristic \\
DSSAE & Deep stacked sparse autoencoder & UC & Unit circle \\
FMI & Fowlkes-Mallows index & ZM & Zernike moment \\
\hline
\end{tabular}

Later, pseudo Zernike moment (PZM) is derived from Zernike moment. PZMs have been proven to give better performances than other moment functions such as $\mathrm{Hu}$ moments, Zernike moments, etc. For example, for an order $p$, there are $(p+1)^{2}$ linearly independent pseudo-Zernike polynomials of orders $\leq p$, while there are only $\frac{1}{2}(p+1)(p+2)$ Zernike polynomials. Hence, PZM is more expressive and offers more feature vectors than $\mathrm{ZM}$.

The kernel of PZMs is a set of orthogonal pseudo-Zernike polynomials defined over the polar coordinate inside a unit circle (UC). The 2D PZM of order $p$ with repetition $q$ of an image $g(r, \theta)$ is defined as [26]

$Z_{p q}=\frac{p+1}{\pi} \int_{-\pi}^{\pi} \int_{0}^{1} W_{p q}^{*}(r, \theta) g(r, \theta) r \mathrm{~d} r \mathrm{~d} \theta$

where the pseudo-Zernike polynomials $W_{p q}(r, \theta)$ of order $p$ are defined as

$$
\begin{aligned}
& W_{p q}(r, \theta)=R_{p q}(r) e^{j q \theta}, \quad j=\sqrt{-1} \\
& R_{p q}(r)=\sum_{k=0}^{p-|q|}(-1)^{k} \frac{(2 p+1-k) !}{k !(p+|q|+1-k) !(p-|q|-k) !} r^{p-k}
\end{aligned}
$$

where $0 \leq|q| \leq p$. In practice, pseudo Zernike functions (https://www.mathworks.com/matlabcentral/ fileexchange/33644-pseudo-zernike-functions) are used for simplicity and fast calculation. Fig. 3 displays pseudo Zernike functions of orders $p \leq 5$.

Note that PZM are defined in terms of polar coordinates $(r, \theta)$ with $|r| \leq 1$. Therefore, the computation of PZM requires a linear transformation of the image plane (IP) coordinates $(w, h), 1 \leq w \leq W, 1 \leq h \leq H$ to the $\mathrm{UC}$ domain $(x, y) \in \mathbb{R}^{2}$. There are two commonly used transformations as shown in Fig. 4: (i) IP over UC; and (ii) IP inside UC. In this study, we use 
the former (IP over UC), because the lesions will not occur within the four corners of the CCT image.

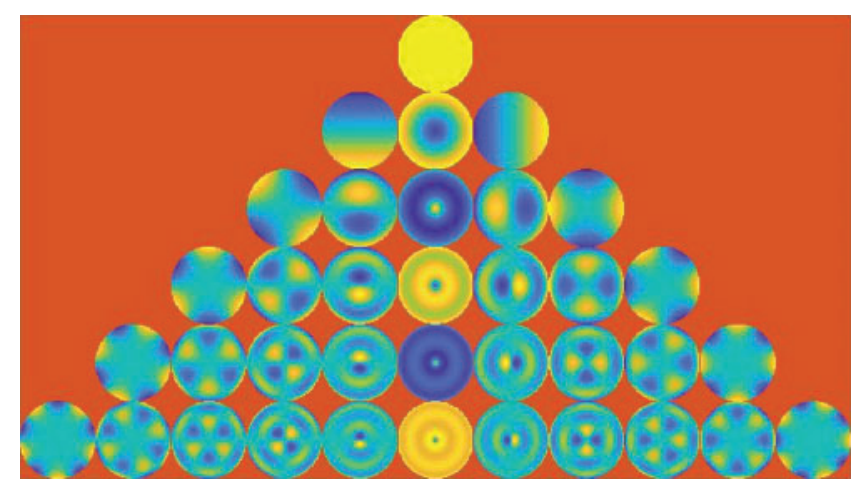

Figure 3: Pseudo Zernike functions of orders $\boldsymbol{p} \leq \mathbf{5}$

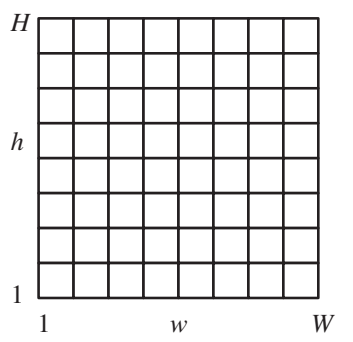

(a)

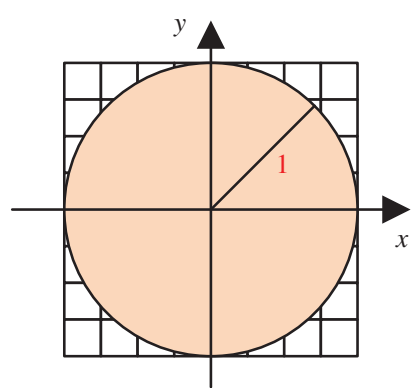

(b)

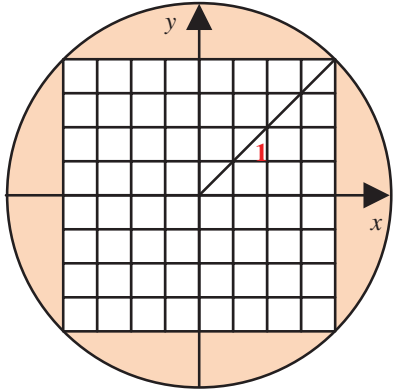

(c)

Figure 4: Two transformation (IP: image plane; UC: unit circle) (a) Raw image plane $W \times H$ (b) IP over UC (c) IP inside UC

\subsection{Autoencoder}

Traditionally, $p$-order PZMs are sent into shallow classifiers, such as multi-layer perceptron [27], adaptive differential evolution wavelet neural network (Ada-DEWNN) [28], linear regression classifier (LRC) [29], kernel support vector machine (KSVM) [30]. In this study, we introduced a customized deep-stacked sparse autoencoder (DSSAE). DSSAE is a type of deep neural network technologies, and we expect DSSAE to achieve better performances than shallow models.

The fundamental element of DSSAE in the autoencoder (AE), which is a typical shallow neural network that learns to map its input $X$ to output $Y$. There is an internal code output $I_{N}$ That represents the input $X$. The whole AE can be divided into two parts: An encoder part $\left(A_{X}, B_{X}\right)$ that maps the input $X$ to the code $I_{N}$, and a decoder part $\left(A_{Y}, B_{Y}\right)$ that maps the code to a reconstructed data $Y$. 
The structure of $\mathrm{AE}$ is displayed in Fig. 5, where the encoder part is with weight $A_{X}$ and bias $B_{X}$, and the decoder part is with weights $A_{Y}$ and bias $B_{Y}$. We have

$I_{N}=z_{L S}\left(A_{X} X+B_{X}\right)$,

$Y=z_{L S}\left(A_{Y} I_{N}+B_{Y}\right)$,

where the output $Y$ is an estimate of input $X$, and $z_{L S}$ is the log sigmoid function

$z_{L S}(x)=\frac{1}{1+\exp (-x)}$.

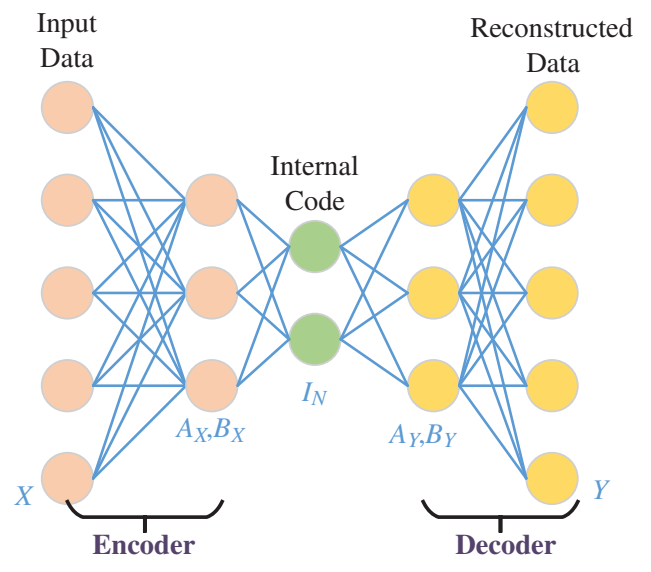

Figure 5: Structure of an $\mathrm{AE}$

\subsection{Sparse Autoencoder}

The sparse autoencoder (SAE) is a variant of AE. SAE encourages sparsity into AE. SAE only allows a small fraction of the hidden neurons to be active at the same time. To minimize the error between the input vector $X$ and the output $Y$, the raw loss function $J_{b}$ of $\mathrm{AE}$ is deduced as:

$J_{b}\left(A_{X}, A_{Y}, B_{X}, B_{Y}\right)=\frac{1}{N_{S}}\|Y-X\|^{2}$,

where $N_{S}$ means the number of training samples. From Eqs. (4) and (5), we find the output $Y$ can be expressed in the way of

$Y=z_{A E}\left(X \mid A_{X}, A_{Y}, B_{X}, B_{Y}\right)$,

where $z_{A E}$ is the abstract of AE function [31]. Hence, Eq. (7) can be revised as

$J_{b}\left(A_{X}, A_{Y}, B_{X}, B_{Y}\right)=\frac{1}{N_{S}}\left\|z_{A E}\left(X \mid A_{X}, A_{Y}, B_{X}, B_{Y}\right)-X\right\|^{2}$. 
To avoid over-complete mapping or learn a trivial mapping, we define one $L_{2}$ regularization term $\Gamma_{A}$ of the weights $\left(A_{X}, A_{Y}\right)$ and one regularization term $\Gamma_{S}$ of the sparsity constraint. Therefore, the loss function $J_{l}$ of $\mathrm{SAE}$ is derived as:

$J_{l}\left(A_{X}, A_{Y}, B_{X}, B_{Y}\right)=\frac{1}{N_{S}}\left\|z_{A E}\left(X \mid A_{X}, A_{Y}, B_{X}, B_{Y}\right)-X\right\|^{2}+a_{S} \times \Gamma_{S}+a_{A} \times \Gamma_{A}$,

where $a_{S}$ stands for the sparsity regulation factor, and $a_{A}$ the weight regulation factor. The sparsity regularization term $\Gamma_{s}$ is defined as:

$\Gamma_{s}=\sum_{m=1}^{\left|I_{N}\right|} z_{K L}\left(\rho, \hat{\rho}_{m}\right)=\sum_{m=1}^{\left|I_{N}\right|} \rho \log \frac{\rho}{\hat{\rho}_{m}}+(1-\rho) \log \frac{1-\rho}{1-\hat{\rho}_{m}}$,

where $z_{K L}$ stands for the Kullback-Leibler divergence [32] function, $\left|I_{N}\right|$ is the number of elements of internal code output $I_{N}, \hat{\rho}_{m}$ is the $m$-th neuron's average activation value over all $N_{S}$ training samples, and $\rho$ is its desired value, viz., sparsity proportion factor. The weight regularization term $\Gamma_{A}$ is defined as

$\Gamma_{A}=\frac{1}{2} \times\left\|A_{X} \quad A_{Y}\right\|_{2}^{2}$.

The training procedure is set to scaled conjugate gradient descent (SCGD) method.

\subsection{Deep Stacked Sparse Autoencoder}

We use SAE as the building block and establish the final deep-stacked sparse autoencoder (DSSAE) classifier by following three operations: (i) We include input layer, preprocessing layer, PZM layer; (ii) We stack four SAEs; (iii) We append softmax layer at the bottom of our AI model. The details of this proposed PZM-DSSAE model are listed in Tab. 2 and illustrated in Fig. 6. After processing, all the CCT images are normalized to fixed grayscaled images with the size of $W \times H$. Then, PZM is applied to obtain feature vector with size of $(p+1)^{2} \times 1$. In the classification stage, four SAE blocks with number of neurons of $\left(M_{1}, M_{2}, M_{3}, M_{4}\right)$ are employed. Finally, a softmax layer with neurons of $M_{c}$ is appended, where $M_{c}$ means the number of categories to be identified.

Table 2: Layer details of proposed PZM-DSSAE model

\begin{tabular}{lll}
\hline Layer & Trainable weights & Size \\
\hline Input & None & $1024 \times 1024 \times 3$ \\
Preprocessing & None & $W \times H$ \\
PZM & None & $(p+1)^{2} \times 1$ \\
1st SAE & $(p+1)^{2} \times M_{1}$ & $M_{1}$ \\
2nd SAE & $M_{1} \times M_{2}$ & $M_{2}$ \\
3rd SAE & $M_{2} \times M_{3}$ & $M_{3}$ \\
4th SAE & $M_{3} \times M_{4}$ & $M_{4}$ \\
Softmax & $M_{4} \times M_{c}$ & $M_{c} \times 1$ \\
\hline
\end{tabular}



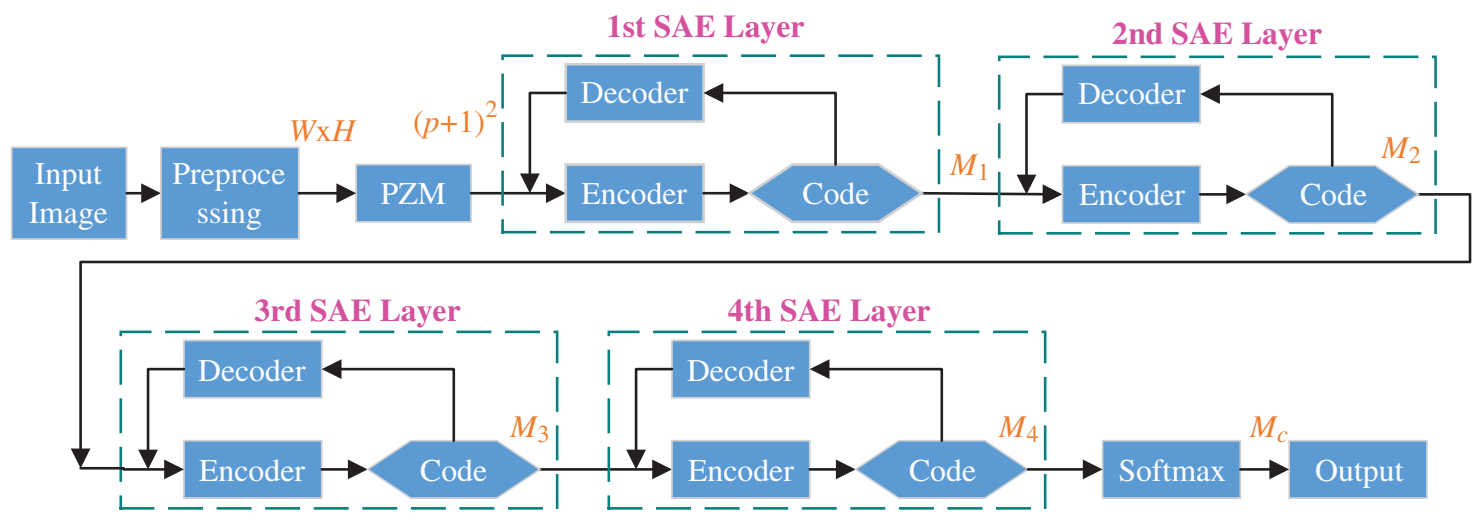

Figure 6: Structure of proposed PZM-DSSAE model

\subsection{8-Way Data Augmentation}

The small size of training images causes overfitting, one solution to data augmentation (DA) that creates fake training images. Multiple-way DA (MDA) is an enhanced method of DA. Wang [33] proposed a 14-way data augmentation, in which they employed seven different DA techniques on $k$-th training image $g(k)$ and its mirrored image $g^{(m)}(k)$.

In this study, we add two new DA techniques, speckle noise (SN) [34] and salt-and-pepper noise (SAPN). SN altered image is defined as

$g^{S N}(k)=g(k)+N_{S N} * g(k)$,

where $N_{S N}$ is uniformly distributed random noise. The mean and variance of $N_{S N}$ is set to $m_{S N}$ and $v_{S N}$, respectively.

For the $k$-th training image $g(k)$, the SAPN altered image [35] is defined as $g^{S A P N}(k)$ with its values are set as

$$
\left\{\begin{array}{l}
\mathrm{P}\left(g^{S A P N}=g\right)=1-\gamma_{d}^{\text {sapn }}, \\
\mathrm{P}\left(g^{S A P N}=g_{\min }\right)=\frac{\gamma_{d}^{\text {sapn }}}{2}, \\
\mathrm{P}\left(g^{S A P N}=g_{\max }\right)=\frac{\gamma_{d}^{\text {sapn }}}{2},
\end{array}\right.
$$

where $\gamma_{d}^{\text {sapn }}$ stands for noise density, and $\mathrm{P}$ the probability function. $g_{\min }$ and $g_{\max }$ correspond to black and white colors, respectively. The definitions of $g_{\min }$ and $g_{\max }$ can be found in Algorithm 1.

First, $Q^{D}$ different DA methods as shown in Fig. 7 are applied to $g(k)$. Let $\mathbf{H}_{m}, m=$ $1, \ldots, Q^{D}$ denote each DA operation, we have the augmented dataset on raw image $g(k)$ as:

$\mathbf{H}_{m}[g(k)], \quad m=1, \ldots, Q^{D}$. 


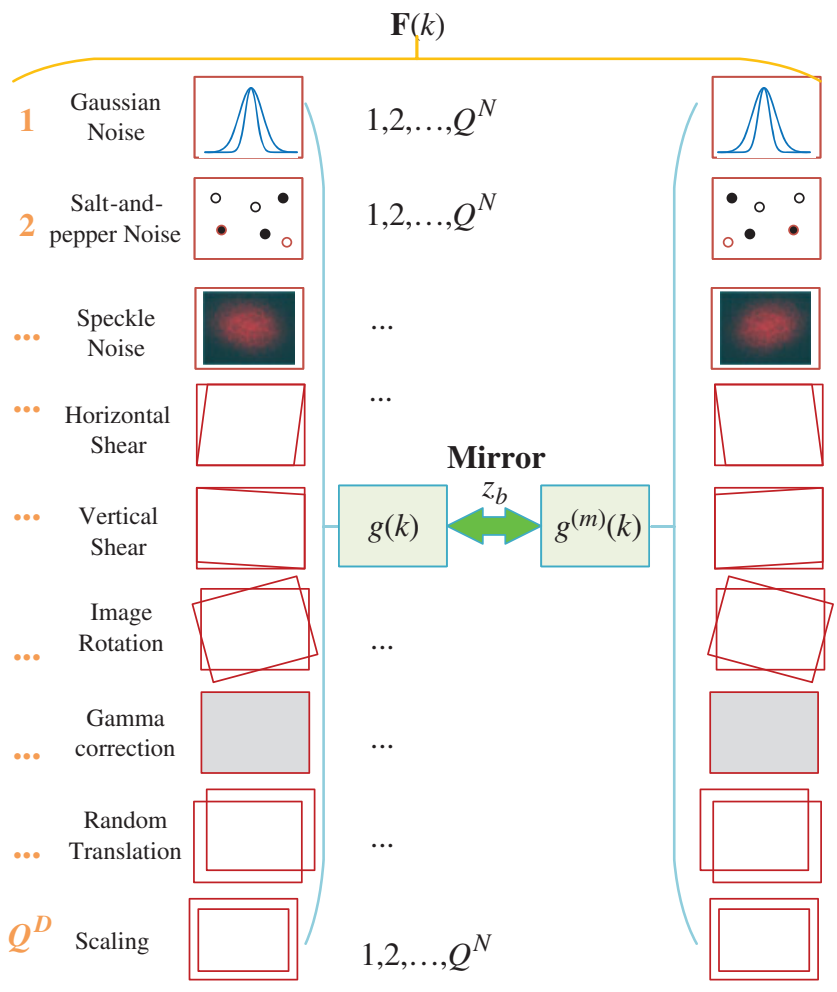

Figure 7: Diagram of proposed 16-way DA

Suppose $Q^{N}$ stands for the size of generated new images for each DA method, we have $\left|\mathbf{H}_{m}[g(k)]\right|=Q^{N}$.

Second, horizontal mirrored image is generated as:

$g^{(m)}(k)=z_{b}[g(k)]$

where $z_{b}$ stands for horizontal mirror function.

Third, all the $Q^{D}$ different DA methods are performed on the mirror image $p_{c}(k)$, and generate $Q^{D}$ different dataset.

$\begin{cases}\mathbf{H}_{m}\left[g^{(m)}(k)\right], & m=1, \ldots, Q^{D} \\ \left|\mathbf{H}_{m}\left[g^{(m)}(k)\right]\right|=Q^{N}, & m=1, \ldots, Q^{D}\end{cases}$

Fourth, the raw image $g(k)$, the mirrored image $g^{(m)}(k)$, all the above $Q^{D}$-way results of raw image $\mathbf{H}_{m}[g(k)]$, and $Q^{D}$-way DA results of horizontal mirrored image $\mathbf{H}_{m}\left[g^{(m)}(k)\right]$, are 
combined together. The final generated dataset from $g(k)$ is defined as $\mathbf{F}(k)$ :

$$
g(k) \mapsto \mathbf{F}(k)=z_{a}\left\{\begin{array}{ll}
\underbrace{}_{Q^{N}} \underbrace{\mathbf{H}_{1}[k(k)]}_{Q^{N}} & \underbrace{\mathbf{H}_{1}\left[g^{(m)}(k)\right]}_{Q^{(m)}(k)} \\
\ldots & \ldots \\
\underbrace{\mathbf{H}_{Q^{D}[g(k)]}}_{Q^{N}} & \underbrace{\mathbf{H}_{Q^{D}\left[g^{(m)}(k)\right]}}_{Q^{N}}
\end{array}\right\}
$$

where $z_{a}$ stands for the concatenation function. Suppose augmentation factor is $Q^{A}$, which stands for the number of images in $\mathbf{F}(k)$, we have

$$
Q^{A}=\frac{|\mathbf{F}(k)|}{|g(k)|}=\frac{\left(1+Q^{D} \times Q^{N}\right) \times 2}{1}=2 \times Q^{D} \times Q^{N}+2
$$

Algorithm 2 summarizes the pseudocode of proposed 18-way DA method.

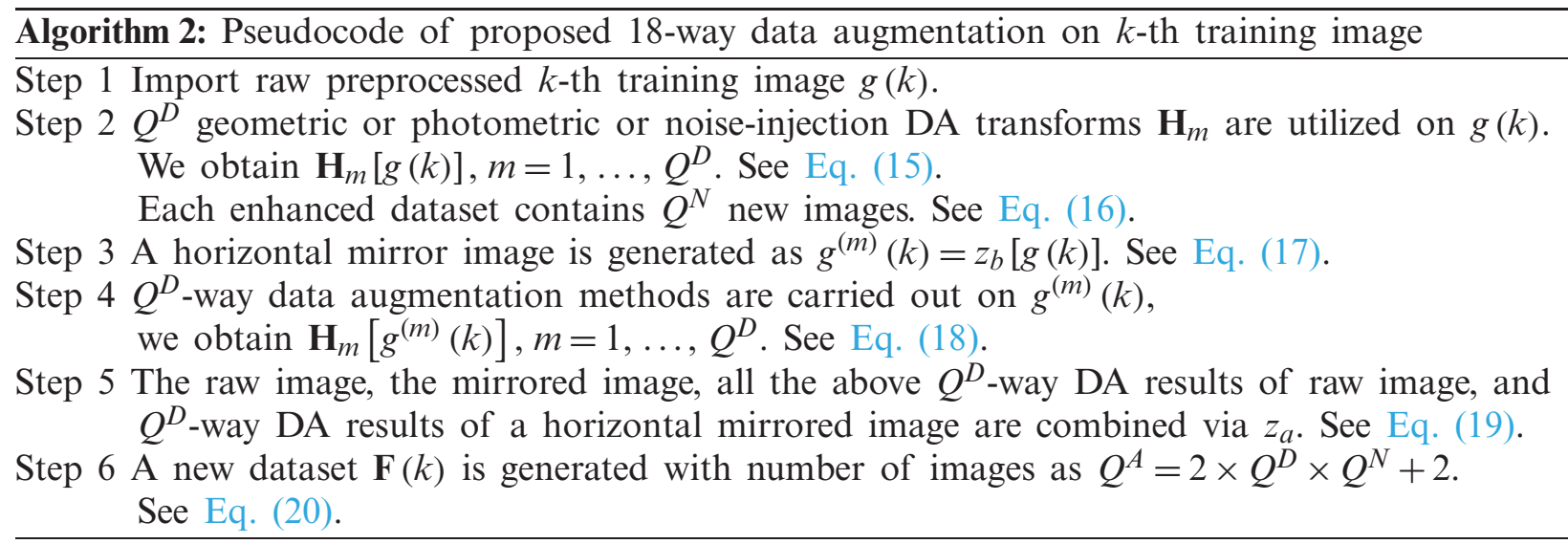

\subsection{Cross-Validation}

$F$-fold cross-validation was used in this study. The whole dataset is divided into $F$ folds. At $f$-th trial, $1 \leq f \leq F$, the $f$-th fold is selected as the test, and the rest $F-1$ folds [36]: $[1, \ldots, f-1, f+1, \ldots, F]$ are selected as training set (Fig. 8). In this study, suppose $F=10$, then each fold will contain 32 COVID-19 images and $32 \mathrm{HC}$ images.

\subsection{Evaluation}

To avoid randomness, we run the whole above procedure $N^{R}$ times with different initial random seeds and different cross-validation partitions. The ideal confusion matrix $(\mathrm{CM}) R^{\text {ideal }}$ is defined as

$R^{\text {ideal }}=\left\{r^{\text {ideal }}(m, n)\right\}=N^{R} \times\left[\begin{array}{ll}C_{1} & 0 \\ 0 & C_{2}\end{array}\right]$, 


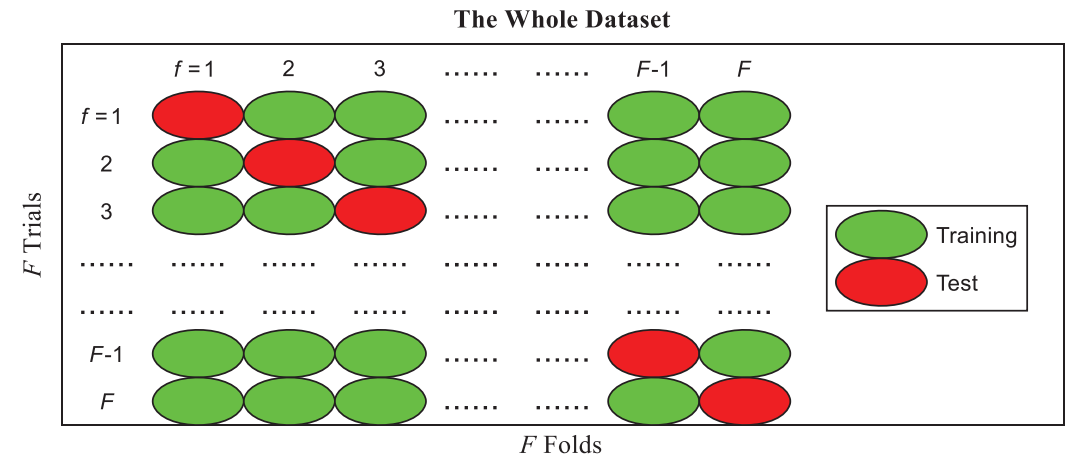

Figure 8: $F$-fold cross validation

Note here the off-diagonal entries of $R^{\text {ideal }}$ are all zero, viz., $r^{\text {ideal }}(m, n)=0, \forall m \neq n$. $C_{1}$ and $C_{2}$ are the number of samples of each category, which can be found in Algorithm 1. Seven measures are defined based on realistic CM [37] defined as:

$R^{\text {real }}=\left\{r^{\text {real }}(m, n)\right\}=\left[\begin{array}{cc}T P & F N \\ F P & T N\end{array}\right]$,

The first four measures are sensitivity, specificity, precision and accuracy, common in most pattern recognition papers. The last three measures are F1 score, Matthews correlation coefficient (MCC) [38], and Fowlkes-Mallows index (FMI) [39]. They are defined as:

$$
\left\{\begin{array}{l}
F 1=\frac{2 T P}{2 T P+F P+F N} \\
M C C=\frac{T P \times T N-F P \times F N}{\sqrt{(T P+F P) \times(T P+F N) \times(T N+F P) \times(T N+F N)}}, \\
F M I=\sqrt{\frac{T P}{T P+F P} \times \frac{T P}{T P+F N}}
\end{array}\right.
$$

Besides, the receiver operating characteristic (ROC) curve [40] is used to provide a graphical plot of our model. ROC curve is created by plotting the true positive rate against the false-positive rate at various threshold settings. The area under the curve (AUC) is also calculated.

\section{Experimental Results}

\subsection{Parameter Setting}

Tab. 3 displays the parameter setting of this study. The number of samples of each class is 320. The minimum and maximum grayscale values are set to $(0,255)$. For the crop operation, 200 pixels are removed from all four sides. The preprocessed image is with size of $256 \times 256$. The max order of PZM is set to 19 , so we have $(19+1)^{2}=400$ PZM features. The weight regularization factor $a_{A}=0.001$, the sparsity regulation factor $a_{S}=1.1$, and the sparsity proportion factor is $\rho=0.05$. The neurons of four SAEs are 300, 200,100, and 50, respectively. The number of classes to be classified is set to 2. The number of folds in cross-validation is set to 10 . The mean and variance of uniformly distributed random noise in $\mathrm{SN}$ are set to 0 and 0.05 , respectively. The noise 
density of SAPN is set to 0.05 . The number of different DA methods is set to 9 , and the number of the newly generated image is set to 30 . The augmentation factor is obtained as $Q^{A}=542$ (See Algorithm 2). The number of runs is set to 10 .

Table 3: Parameter setting

\begin{tabular}{llllll}
\hline Parameter & Value & Parameter & Value & Parameter & Value \\
\hline$\left(C_{1}, C_{2}\right)$ & $(320,320)$ & $\rho$ & 0.05 & $m_{S N}$ & 0 \\
$\left(g_{\min }, g_{\max }\right)$ & $(0,255)$ & $M_{1}$ & 300 & $v_{S N}$ & 0.05 \\
$\left(m_{1}^{c}, m_{2}^{c}, m_{3}^{c}, m_{4}^{c}\right)$ & $(200,200,200,200)$ & $M_{2}$ & 200 & $\gamma_{d}^{\text {sapn }}$ & 0.05 \\
$(W, H)$ & $(256,256)$ & $M_{3}$ & 100 & $Q^{D}$ & 9 \\
$p$ & 19 & $M_{4}$ & 50 & $Q^{N}$ & 30 \\
$a_{A}$ & 0.001 & $M_{c}$ & 2 & $Q^{A}$ & 542 \\
$a_{S}$ & 1.1 & $F$ & 10 & $N^{R}$ & 10 \\
\hline
\end{tabular}

\subsection{Illustration of 18-Way Data Augmentation}

Fig. 9 shows the $Q^{D}$-way DA to the raw image. Due to the page limit, the mirrored image and its corresponding DA results are not displayed. As can be observed in Fig. 9, the multiple-way DA can increase our training images' diversity.

\subsection{Statistical Analysis and Transformation Comparison}

Tab. 4 gives the 10 runs of 10 -fold cross-validation, where we can see our method achieves a sensitivity of $92.06 \% \pm 1.54 \%$, a specificity of $92.56 \% \pm 1.06 \%$, a precision of $92.53 \% \pm 1.03 \%$, and an accuracy of $92.31 \% \pm 1.08 \%$. Its F1 score, MCC, and FMI arrive at $92.29 \% \pm 1.10 \%$, $84.64 \% \pm 2.15 \%$, and $92.29 \% \pm 1.10 \%$, respectively. The AUC is 0.9576 .

In addition, we compared the two transformation settings: IP over UC against IP inside UC (See Fig. 4). The IP inside the UC setting achieves a sensitivity of $91.84 \% \pm 2.18 \%$, a specificity of $92.44 \% \pm 1.31 \%$, and an accuracy of $92.14 \% \pm 1.12 \%$, which are worse than IP over UC setting. This comparison result demonstrates the reason why we choose IP over UC in this study. Particularly, the receiver operating characteristics (ROC) curves of both settings are displayed in Fig. 10.

\subsection{Comparison to State-of-the-Art Methods}

This proposed PZM-DSSAE method is compared with 8 state-of-the-art methods. The comparison results are carried out on the same dataset via 10 runs of 10 -fold cross-validation, and the results are displayed in Tab. 5. Fig. 11 displays the error bar of the proposed method against 8 state-of-the-art methods. We can see that the proposed PZM-DSSAE gives the best performance among all the methods. The reason is three folds: (i) We try to use PZM as the feature descriptors, (ii) DSSAE is used as the classifier, (iii) 18-way DA is employed to solve the overfitting problem. 


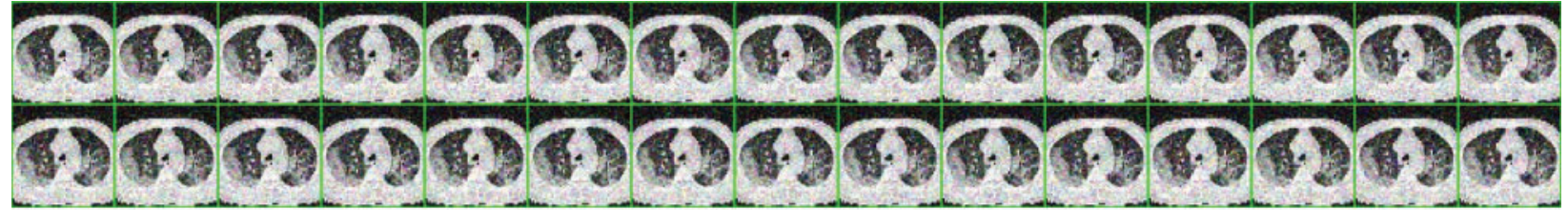

(a)

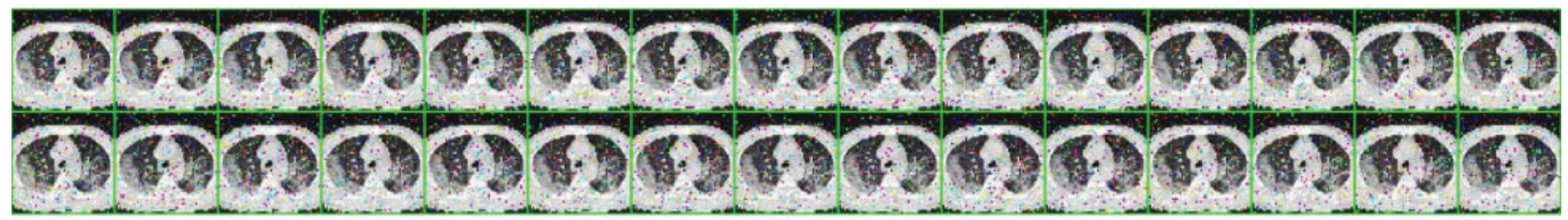

(b)

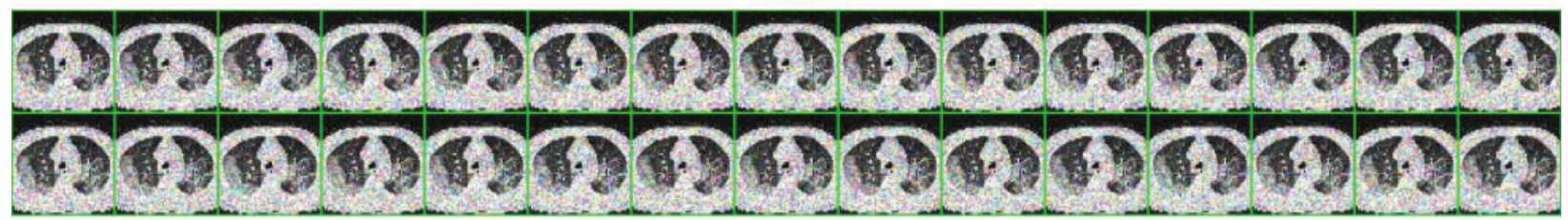

(c)

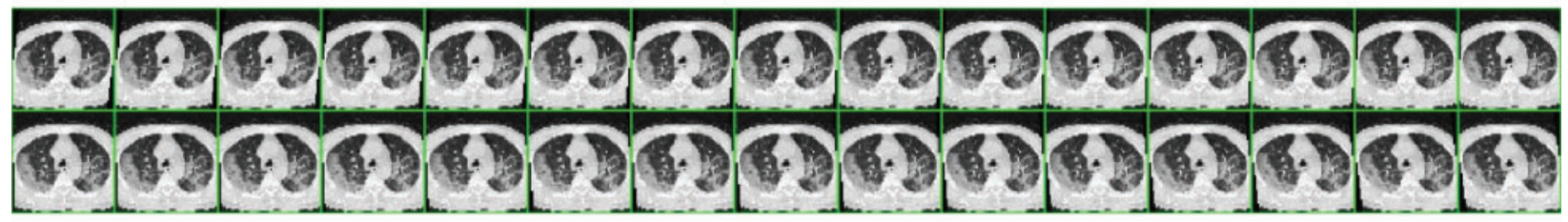

(d)

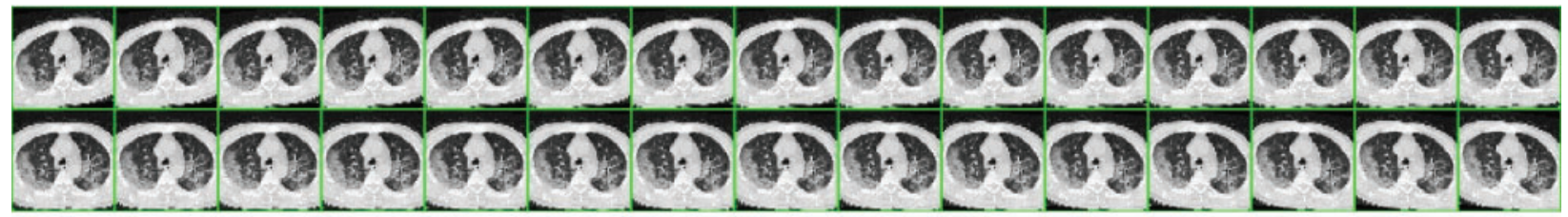

(e)

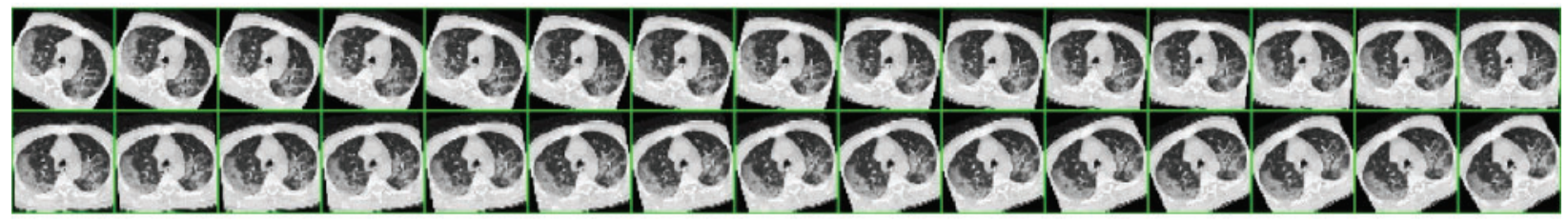

(f)

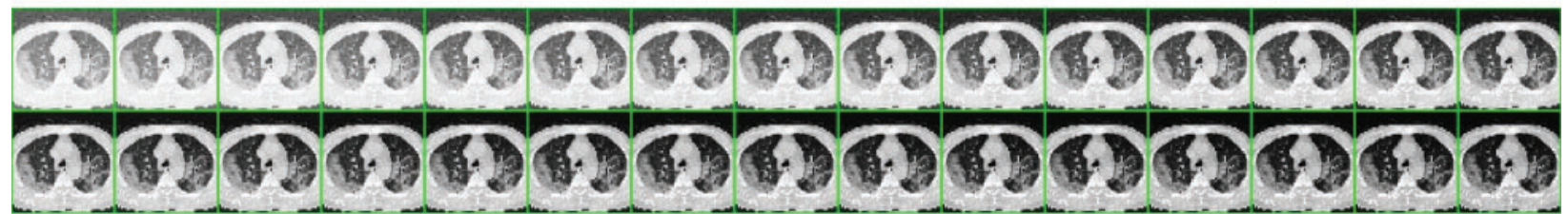




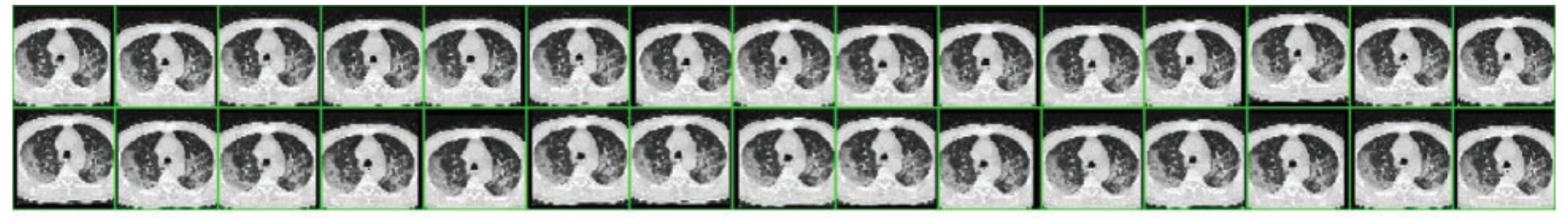

(h)

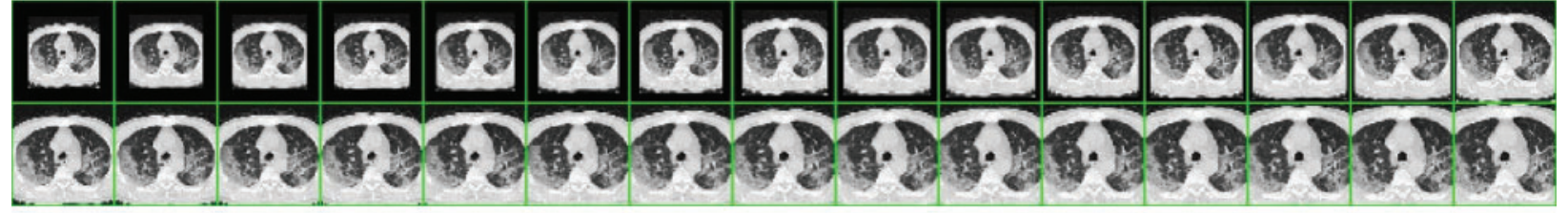

(i)

Figure 9: $\boldsymbol{Q}^{\boldsymbol{D}}$-way DA results of raw image (a) Gaussian noise (b) SAPN (c) SN (d) Horizontal shear (e) Vertical shear (f) Rotation (g) Gamma correction (h) Random translation (i) Scaling

Table 4: 10 Runs of statistical analysis of proposed PZM-DSSAE method

\begin{tabular}{llllllll}
\hline Run & Sen & Spc & Prc & Acc & F1 & MCC & FMI \\
\hline 1 & 90.94 & 91.88 & 91.80 & 91.41 & 91.37 & 82.82 & 91.37 \\
2 & 91.56 & 91.56 & 91.56 & 91.56 & 91.56 & 83.13 & 91.56 \\
3 & 91.88 & 92.50 & 92.45 & 92.19 & 92.16 & 84.38 & 92.16 \\
4 & 92.19 & 91.56 & 91.61 & 91.88 & 91.90 & 83.75 & 91.90 \\
5 & 91.25 & 92.19 & 92.11 & 91.72 & 91.68 & 83.44 & 91.68 \\
6 & 90.63 & 92.19 & 92.06 & 91.41 & 91.34 & 82.82 & 91.34 \\
7 & 93.75 & 93.13 & 93.17 & 93.44 & 93.46 & 86.88 & 93.46 \\
8 & 95.31 & 94.38 & 94.43 & 94.84 & 94.87 & 89.69 & 94.87 \\
9 & 92.81 & 91.88 & 91.95 & 92.34 & 92.38 & 84.69 & 92.38 \\
10 & 90.31 & 94.38 & 94.14 & 92.34 & 92.19 & 84.76 & 92.20 \\
MSD & $92.06 \pm 1.54$ & $92.56 \pm 1.06$ & $92.53 \pm 1.03$ & $92.31 \pm 1.08$ & $92.29 \pm 1.10$ & $84.64 \pm 2.15$ & $92.29 \pm 1.10$
\end{tabular}

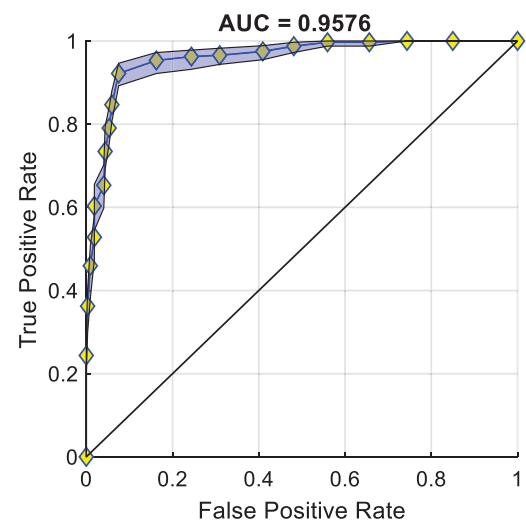

(a)

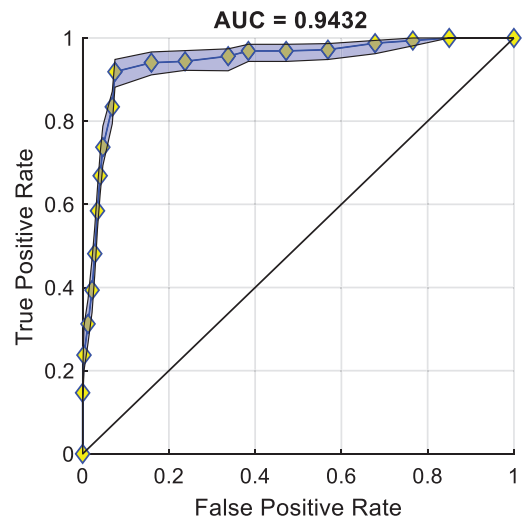

(b)

Figure 10: ROC curves of two settings (a) IP over UC (b) IP inside UC 
Table 5: Comparison to state-of-the-art methods

\begin{tabular}{|c|c|c|c|c|c|c|c|}
\hline Method & Sen & $\mathrm{Spc}$ & Prc & Acc & F1 & $\mathrm{MCC}$ & FMI \\
\hline & & & & & & & $83.32 \pm 1.36$ \\
\hline & & & & & & & \\
\hline & & & & & & & \\
\hline & & & & & & & \\
\hline$t$ [15] & & & & & & & \\
\hline & & & & & & & \\
\hline & & & & & & & \\
\hline GLCMSVM [18] & $72.38 \pm 2.68$ & $77.38 \pm 1.96$ & $76.22 \pm 1.21$ & $74.88 \pm 0$ & $74.21 \pm 1.25$ & $49.85 \pm 1.70$ & $74.25 \pm 1.21$ \\
\hline PZM-DSSAE (Ours) & $92.06 \pm 1.54$ & $92.56 \pm 1.06$ & $92.53 \pm 1.03$ & $92.31 \pm 1.08$ & $92.29 \pm 1.10$ & $84.64 \pm 2.15$ & $92.29 \pm 1.10$ \\
\hline
\end{tabular}

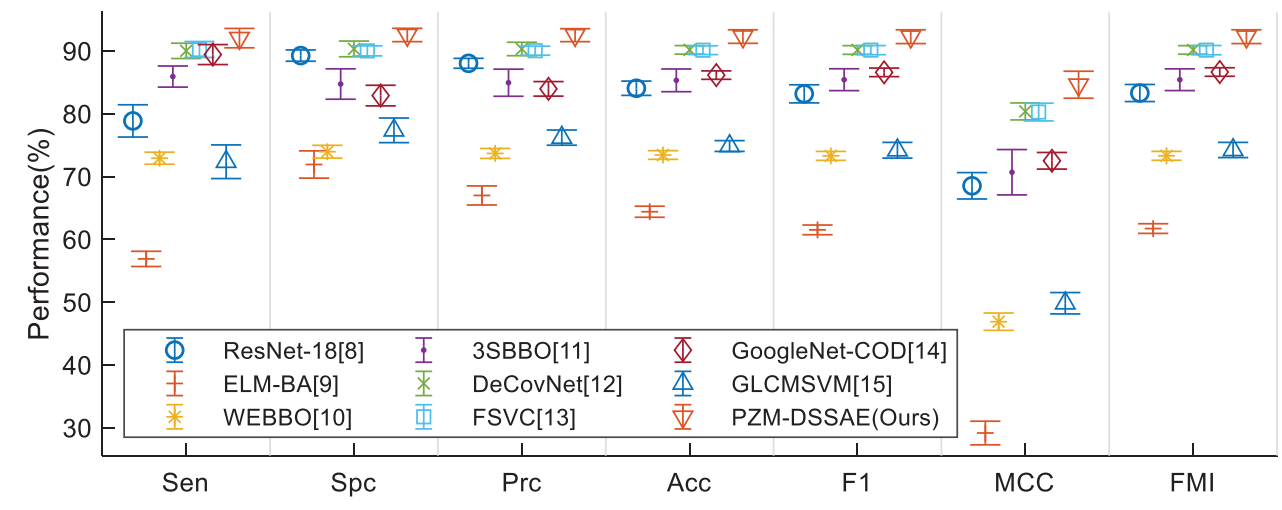

Figure 11: Error bar plot of method comparison

\section{Conclusion}

This study proposed a novel PZM-DSSAE system for COVID-19 diagnosis. As far as the authors' best known, we are the first to apply PZM to COVID-19 image analysis. Also, two other improvements are carried out: (i) DSSAE is used as the classifier, and (ii) multiple-way data augmentation is employed to generalize the classifier. Our model yields a sensitivity of $92.06 \% \pm$ $1.54 \%$, a specificity of $92.56 \% \pm 1.06 \%$, an accuracy of $92.31 \% \pm 1.08 \%$, and an AUC of 0.9576 .

In the future, we shall collect more COVID-19 images from more patients and multiple modalities. Also, other advanced AI models will be tested, such as graph neural networks and attention networks.

Funding Statement: This study was supported by Royal Society International Exchanges Cost Share Award, UK (RP202G0230); Medical Research Council Confidence in Concept Award, UK (MC_PC_17171); Hope Foundation for Cancer Research, UK (RM60G0680); Global Challenges Research Fund (GCRF), UK (P202PF11)

Conflicts of Interest: The authors declare that they have no conflicts of interest to report regarding the present study. 


\section{References}

[1] B. Zemrani, M. Gehri, E. Masserey, C. Knob and R. Pellaton, "A hidden side of the COVID-19 pandemic in children: The double burden of undernutrition and overnutrition," International Journal for Equity in Health, vol. 20, no. 1, pp. 4, 2021.

[2] M. Bassi, L. Negri, A. Delle Fave and R. Accardi, "The relationship between post-traumatic stress and positive mental health symptoms among health workers during COVID-19 pandemic in Lombardy," Italy Journal of Affective Disorders, vol. 280, pp. 1-6, 2021.

[3] D. Simiies, A. R. Stengaard, L. Combs, D. Raben and T. C. I. A. Euro, "Impact of the COVID-19 pandemic on testing services for HIV, viral hepatitis and sexually transmitted infections in the WHO European Region, march to august 2020," Eurosurveillance, vol. 25, pp. 7, 2020.

[4] F. Salahshour, M.-M. Mehrabinejad, M. N. Toosi, M. Gity, H. Ghanaati et al., "Clinical and chest CT features as a predictive tool for COVID-19 clinical progress: Introducing a novel semi-quantitative scoring system," European Radiology, vol. 11, pp. 1-11, 2021.

[5] A. M. Ismael and A. Sengur, "The investigation of multiresolution approaches for chest X-ray image based COVID-19 detection," Health Information Science and Systems, vol. 8, no. 1, pp. 1-13, 2020.

[6] A. Hata, M. Yanagawa, Y. Yoshida, T. Miyata, N. Kikuchi et al., "The image quality of deep-learning image reconstruction of chest CT images on a mediastinal window setting," Clinical Radiology, vol. 76, no. 2, pp. 9, 2021.

[7] Y. Satoh, U. Motosugi, M. Imai, Y. Omiya and H. Onishi, "Evaluation of image quality at the detector's edge of dedicated breast positron emission tomography," Ejnmmi Physics, vol. 8, no. 1, pp. 14, 2021.

[8] M. A. Khan, S. Kadry, Y.-D. Zhang, T. Akram, M. Sharif et al., "Prediction of COVID-19-pneumonia based on selected deep features and one class kernel extreme learning machine," Computers \& Electrical Engineering, vol. 90, pp. 106960, 2021.

[9] T. Akram, M. Attique, S. Gul, A. Shahzad, M. Altaf et al., "A novel framework for rapid diagnosis of COVID-19 on computed tomography scans," Pattern Analysis and Applications, pp. 1-14, 2021. https://doi.org/10.1007/s10044-020-00950-0.

[10] H. T. Rauf, M. I. U. Lali, M. A. Khan, S. Kadry, H. Alolaiyan et al., "Time series forecasting of COVID-19 transmission in Asia Pacific countries using deep neural networks," Personal and Ubiquitous Computing, vol. 8, pp. 1-18, 2021.

[11] M. H. Guo and Y. Z. Du, "Classification of thyroid ultrasound standard plane images using ResNet-18 networks," in IEEE 13th Int. Conf. on Anti-Counterfeiting, Security, and Identification, Xiamen, China, pp. 324-328, 2019.

[12] S. Lu, "A pathological brain detection system based on extreme learning machine optimized by Bat algorithm," CNS \& Neurological Disorders-Drug Targets, vol. 16, no. 1, pp. 23-29, 2017.

[13] X. Yao, "COVID-19 detection via wavelet entropy and biogeography-based optimization," in COVID-19: Prediction, Decision-Making, and Its Impacts, K. C. Santosh, A. Joshi (Eds.), Cham: Springer, pp. 69-76, 2020.

[14] X. Wu, "Diagnosis of COVID-19 by wavelet renyi entropy and three-segment biogeography-based optimization," International Journal of Computational Intelligence Systems, vol. 13, no. 1, pp. 13321344, 2020.

[15] X. G. Wang, X. B. Deng, Q. Fu, Q. Zhou, J. P. Feng et al., "A weakly-supervised framework for COVID-19 classification and lesion localization from chest CT," IEEE Transactions on Medical Imaging, vol. 39, no. 8, pp. 2615-2625, 2020.

[16] E. S. M. El-kenawy, A. Ibrahim, S. Mirjalili, M. M. Eid and S. E. Hussein, "Novel feature selection and voting classifier algorithms for COVID-19 classification in CT images," IEEE Access, vol. 8, pp. 179317$179335,2020$.

[17] X. Yu, S.-H. Wang, X. Zhang and Y.-D. Zhang, "Detection of COVID-19 by GoogLeNet-COD," in Int. Conf. on Intelligent Computing, Bari, Italy, pp. 499-509, 2020. 
[18] Y. Chen, "Covid-19 classification based on gray-level co-occurrence matrix and support vector machine," in COVID-19: Prediction, Decision-Making, and Its Impacts, K. C. Santosh, A. Joshi (Eds.), Singapore: Springer, Cham, pp. 47-55, 2020.

[19] M. A. Khan, N. Hussain, A. Majid, M. Alhaisoni, S. A. C. Bukhari et al., "Classification of positive COVID-19 CT scans using deep learning," Computers, Materials and Continua, vol. 66, no. 2, pp. 1$15,2021$.

[20] Y. D. Zhang, "A seven-layer convolutional neural network for chest CT based COVID-19 diagnosis using stochastic pooling," IEEE Sensors Journal, vol. 1, pp. 1, 2021.

[21] M.-K. Hu, "Visual pattern recognition by moment invariants," IRE Transactions on Information Theory, vol. 8, pp. 179-187, 1962.

[22] S. S. Gornale, P. U. Patravali and P. S. Hiremath, "Automatic detection and classification of knee osteoarthritis using Hu's invariant moments," Frontiers in Robotics and AI, vol. 7, pp. 8, 2020.

[23] S. Ouchtati, A. Chergui, S. Mavromatis, B. Aissa and J. Sequeira, "Novel method for brain tumor classification based on use of image entropy and seven Hu's invariant moments," Traitement Du Signal, vol. 36, no. 6, pp. 483-491, 2019.

[24] M. R. Teague, "Image analysis via the general theory of moments," Journal Optical Soceity America, vol. 70, no. 8, pp. 920-930, 1980.

[25] A. Grosso and T. Scharf, "Scalar analytical expressions for the field dependence of Zernike polynomials in asymmetric optical systems with circular symmetric surfaces," OSA Continuum, vol. 3, no. 10, pp. 2749-2765, 2020.

[26] C. W. Chong, P. Raveendran and R. Mukundan, "The scale invariants of pseudo-Zernike moments," Pattern Analysis and Applications, vol. 6, no. 3, pp. 176-184, 2003.

[27] N. Borzue, K. Faez and IEEE, "Object contour detecting using pseudo zernike moment and multi-layer perceptron," in 22nd Iranian Conf. on Biomedical Engineering, Tehran, Iran, pp. 304-308, 2015.

[28] S. P. Singh and S. Urooj, "An improved CAD system for breast cancer diagnosis based on generalized pseudo zernike moment and Ada-DEWNN classifier," Journal of Medical Systems, vol. 40, no. 4, pp. 123, 2016.

[29] S. Du, "Alzheimer's disease detection by pseudo zernike moment and linear regression classification," CNS \& Neurological Disorders-Drug Targets, vol. 16, no. 1, pp. 11-15, 2017.

[30] Y. Jiang, "Exploring a smart pathological brain detection method on pseudo Zernike moment," Multimedia Tools and Applications, vol. 77, no. 17, pp. 22589-22604, 2018.

[31] D. K. Mohanty, A. K. Parida and S. Suman, "Financial market prediction under deep learning framework using auto encoder and kernel extreme learning machine," Applied Soft Computing, vol. 99, no. 3, pp. 14, 2021.

[32] J. Lequesne and P. Regnault, "vsgoftest: An R package for goodness-of-fit testing based on KullbackLeibler divergence," Journal of Statistical Software, vol. 96, no. Code Snippet 1, pp. 26, 2020.

[33] S.-H. Wang, "Covid-19 classification by FGCNet with deep feature fusion from graph convolutional network and convolutional neural network," Information Fusion, vol. 67, pp. 208-229, 2021.

[34] C. Buitrago-Duque, R. Castaneda and J. Garcia-Sucerquia, "Pointwise phasor tuning for single-shot speckle noise reduction in phase wave fields," Optics and Lasers in Engineering, vol. 137, pp. 5, 2021.

[35] K. Vasanth and R. Varatharajan, "An adaptive content based closer proximity pixel replacement algorithm for high density salt and pepper noise removal in images," Journal of Ambient Intelligence and Humanized Computing, vol. 3, pp. 1-15, 2020.

[36] D. Muller and F. Kramer, "MIScnn: A framework for medical image segmentation with convolutional neural networks and deep learning," BMC Medical Imaging, vol. 21, pp. 11, 2021.

[37] J. Radoux and P. Bogaert, "About the pitfall of erroneous validation data in the estimation of confusion matrices," Remote Sensing, vol. 12, pp. 23, 2020.

[38] D. Dreizin, F. Goldmann, C. LeBedis, A. Boscak, M. Dattwyler et al., "An automated deep learning method for tile AO/OTA pelvic fracture severity grading from trauma whole-body CT," Journal of Digital Imaging, vol. 21, pp. 1-13, 2021. 
[39] R. Jena, B. Pradhan and A. M. Alamri, "Susceptibility to seismic amplification and earthquake probability estimation using recurrent neural network model in Odisha," India Applied Sciences-Basel, vol. 10, pp. 18, 2020.

[40] A. T. Young, K. Fernandez, J. Pfau, R. Reddy, N. A. Cao et al., "Stress testing reveals gaps in clinic readiness of image-based diagnostic artificial intelligence models," NPJ Digital Medicine, vol. 4, pp. 8, 2021. 